\title{
Electromagnetic wave scattering by a partially coated obstacle in a piecewise homogeneous medium
}

\author{
Yun Chol Kim ${ }^{1}$, Ung Kim${ }^{1}$, and Chol Min $\mathrm{Jo}^{1}$ \\ ${ }^{1}$ Kim Chaek University of Technology
}

May 5, 2020

\begin{abstract}
In this paper, we consider an electromagnetic obstacle scattering problem in a piecewise homogeneous medium. We assume that the incident field is the electromagnetic plane wave and the obstacle consists of metallic and nonmetallic parts. We firstly establish uniqueness and existence for the solution to the electromagnetic obstacle scattering problem. Then we prove that the electric far field pattern satisfies the reciprocity relation and the set of electric far field patterns is complete in a Hilbert space. These results have an important bearing on the solution of the inverse electromagnetic scattering problem i.e. determination of the shape of the obstacle from the knowledge of the electric far field patterns.
\end{abstract}

\section{Hosted file}

Manuscript.doc available at https://authorea.com/users/302445/articles/432524electromagnetic-wave-scattering-by-a-partially-coated-obstacle-in-a-piecewisehomogeneous-medium

\section{Hosted file}

Cover letter.doc available at https://authorea.com/users/302445/articles/432524electromagnetic-wave-scattering-by-a-partially-coated-obstacle-in-a-piecewisehomogeneous-medium

\section{Hosted file}

HighLights.doc available at https://authorea.com/users/302445/articles/432524electromagnetic-wave-scattering-by-a-partially-coated-obstacle-in-a-piecewisehomogeneous-medium 